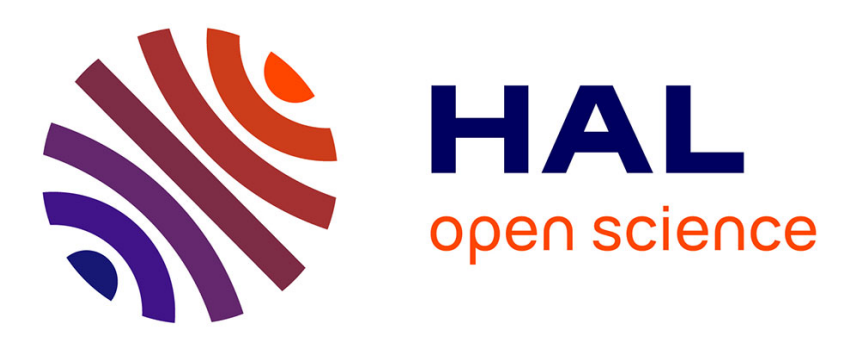

\title{
Solution of the static pair annihilation process in arbitrary dimension
}

\author{
B. Bonnier
}

\section{To cite this version:}

B. Bonnier. Solution of the static pair annihilation process in arbitrary dimension. Physical Review E , 1998, 58 (5), pp.5424-5428. 10.1103/PhysRevE.58.5424 . hal-01566047

\section{HAL Id: hal-01566047 \\ https://hal.science/hal-01566047}

Submitted on 5 Dec 2018

HAL is a multi-disciplinary open access archive for the deposit and dissemination of scientific research documents, whether they are published or not. The documents may come from teaching and research institutions in France or abroad, or from public or private research centers.
L'archive ouverte pluridisciplinaire HAL, est destinée au dépôt et à la diffusion de documents scientifiques de niveau recherche, publiés ou non, émanant des établissements d'enseignement et de recherche français ou étrangers, des laboratoires publics ou privés. 


\title{
Solution of the static pair annihilation process in arbitrary dimension
}

\author{
B. Bonnier* \\ Centre de Physique Théorique et de Modélisation, ESA 5468 du CNRS, Université Bordeaux I, 19 Rue du Solarium, \\ 33174 Gradignan Cedex, France
}

(Received 11 June 1998)

\begin{abstract}
The pair annihilation of identical static particles distributed at random in a $d$-dimensional space is studied in the large time regime for a tunneling law. A superposition approximation is used to close the hierarchy of equations describing the process, and it is shown that the density and pair correlation of surviving particles have universal scaling expressions that are exactly calculated. These results are in close agreement with Monte Carlo simulations in one, two, and three dimensions. [S1063-651X(98)11010-3]
\end{abstract}

PACS number(s): 05.40. $+\mathrm{j}$, 05.70.Ln

\section{INTRODUCTION}

In the static annihilation model (see [1] and references therein), a set of $A$ particles are randomly distributed in a $d$-dimensional space and removed by a fusion reaction $A$ $+A \rightarrow 0$, with isotropic reaction rate $w(r)$, for any pair of particles separated by distance $r$. It must be stressed that the particles $A$ are immobile, the $A+A \rightarrow 0$ reaction corresponding, for example [2], to fusion in triplet states through a tunneling law $w(r)=w_{0} \exp \left(-r / r_{0}\right)$. An interesting aspect of this process at large time is its tendency to self-organization, which is also observed [3,4] for its two species analog $A$ $+B \rightarrow 0$. This property manifests itself through a nontrivial time evolution of the density of surviving particles $\rho(t)$ and of the two-particle correlation function $X(r, t)$. Their behavior is phenomenologically well described in terms of the reaction radius [5] $R(t)$, defined by $t w(R(t)) \simeq 1$, and which is in practice the minimal separation between surviving particles at time $t$. Starting from the initial value $X(r, t=0)$ $=1$, as time increases the correlation vanishes for $r<R(t)$, and when the separation $r$ lies roughly between $R(t)$ and $2 R(t)$, it tends towards a limiting function $X_{\infty}(r)$ quite different from unity, this value being recovered only at larger $r$. The process is thus dominated by fluctuations in the number of particles in a volume of size determined by the reaction radius. It also appears that the density decays as $\rho(t)$ $=C / R^{\alpha}(t)$, where the exponent $\alpha$ is expected [6] to be equal to the space dimensionality $d$. An exact determination in arbitrary dimension of the limiting correlation $X_{\infty}(r)$, which fixes the constant $C$, is the purpose of this work since, to the best of our knowledge, this has not been done, although various numerical or empirical advances have been made.

We achieve this goal by a generalization of the method we have recently applied [7] to solve the $A+A \rightarrow 0$ annihilation process on a one-dimensional lattice for a large class of reaction rates $w(r)$. Our starting point is to consider the evolution equations for the density and for the two-particle correlation function. When the three-particle correlation function appearing in these coupled equations [8] is expressed in terms of the previous functions through a Kirk-

*Electronic address: bonnier@pth.u-bordeaux.fr wood superposition approximation, they reduce to a closed system. Some years ago, such a system was solved numerically, and a comparison of the solutions with the results of Monte Carlo (MC) simulations in various dimensions indicates that the Kirkwood approximation works extremely well $[3,9]$. In one dimension, this approximation is a shielding one, and appears sufficiently accurate to describe the annihilation process at any time, as we have shown in previous work [10]. We thus take this approximation for granted, and we analytically explore its consequences. Our main result is to show that the density and the correlation have, at large time, scaling expressions that involve a unique function $h(x)$, where $x$ is defined as $x=r / R(t)$. In fact, one finds for the density exponent $\alpha=d$, for the density factor $C=h(x$ $=\infty)$ and for the correlation $X(r=x R(t), t)=h(x) / h(\infty)$. The function $h(x)$ is solution of an integral equation explicitly soluble in one dimension, and we recover some of our previous results [7], the other cases being easily solved by a numerical iteration.

In Sec. II we recall the derivation of the evolution equations for the density and for the correlation. Using the superposition approximation we give their leading expression in the large time regime. At this point we introduce a function $\gamma(r, t)$ defined by $\gamma(r, t)=\rho(t) X(r, t)$, since we observe that the previous system implies a simple evolution equation for $\gamma(r, t)$ and that this function suffices to fix the density and the correlation through the relations $\rho(t)=\gamma(\infty, t)$ and $X(r, t)=\gamma(r, t) / \gamma(\infty, t)$, which follow from the normalization condition $X(\infty, t)=1$. Moreover, as we show in Sec. III, the solution of this equation at large time has the scaling form $\gamma(r, t)=h(x) / R^{d}(t)$, provided that $h(x)$ fulfills some conditions. These conditions imply that $h(x)$ vanishes for $x$ $<1$, in agreement with the definition of the reaction radius, that its first nontrivial value at $x=1$ is fixed by the dimensionality, and that its values for $x>1$ obey an integral equation. This equation is solved in Sec. IV for the physical cases $d=1,2,3$ and we check that the solutions are in agreement with the MC data for the density and the correlation function. We summarize our findings in Sec. V.

\section{THE PAIR CORRELATION EVOLUTION EQUATION}

Let $n(r, t)$ be the microscopic particle concentration at time $t$ and position $r, r$ being here a $d$-dimensional vector. 
The ensemble averaged products of these concentrations define the many-center densities $\rho_{m}=\left\langle\Pi_{i=1}^{i=m} n\left(r_{i}, t\right)\right\rangle$. In particular $\langle n(r, t)\rangle=\rho(t)$ and $\rho_{2}=\rho^{2}(t) X\left(\left|r_{1}-r_{2}\right|, t\right)$ where $\rho(t)$ is the global density and $X(r, t)$ the pair correlation function normalized according to $X(r \rightarrow \infty, t)=1$.

Taking into account the annihilation process one can write an infinite system of equations [8] coupling $\rho_{m}$ and $\rho_{m+1}$. For the density one finds

$$
-\frac{d \rho}{d t}(t)=\int w(r) \rho_{2}(r, t) D^{d} r
$$

and considering the decays of a set of two particles $\left(A_{1} A_{2}\right)$ separated by a distance $r$ one obtains the evolution equation of $\rho_{2}(r, t)$. The pair itself can annihilate with a probability $w(r) \rho_{2}(r, t)$, or either member of the pair can annihilate with a third particle $A_{3}$, which happens with a probability $\left(w\left(r^{\prime}\right)+w\left(r^{\prime \prime}\right)\right) \rho_{3}\left(r, r^{\prime}, r^{\prime \prime}\right)$ where $r^{\prime}$ and $r^{\prime \prime}$ are the distances from $A_{3}$ to $A_{1}$ and to $A_{2}$, respectively. Using the symmetry of $\rho_{3}$ one gets

$$
-\frac{\partial \rho_{2}}{\partial t}(r, t)=w(r) \rho_{2}(r, t)+2 \int w\left(r^{\prime}\right) \rho_{3}\left(r, r^{\prime}, r^{\prime \prime}\right) D^{d} r^{\prime},
$$

which becomes an equation for $\rho_{2}$ when one uses the Kirkwood [11] approximation

$$
\rho_{3}\left(r, r^{\prime}, r^{\prime \prime}\right)=\rho^{3}(t) X(r, t) X\left(r^{\prime}, t\right) X\left(r^{\prime \prime}, t\right) .
$$

It is also convenient in Eq. (2) to decompose the $r^{\prime}$ integration region into 3 subdomains $E_{1}, E_{2}$, and $E(r)$ that we describe here in the three-dimensional case, its generalization to other dimensionalities being straightforward. Let $P_{1}$ and $P_{2}$ be those planes perpendicular to the line $A_{1} A_{2}$ and containing the points $A_{1}$ and $A_{2}$, respectively. Then $E(r)$ denotes the region between $P_{1}$ and $P_{2}, E_{1}$ the half-space bounded by $P_{1}$ and where $r^{\prime}<r^{\prime \prime}$, and finally the remaining space is $E_{2}$, where $r^{\prime}>r^{\prime \prime}$. We are now prepared to derive from Eqs. (2) and (3) the leading order expression of $\partial \rho_{2}(r, t) / \partial t$ in the large $r$ and $t$ regime. First of all, the integration domain $E_{2}$ can be neglected due to the exponential decay of the rate $w\left(r^{\prime}\right), r^{\prime}$ being in $E_{2}$ greater than $r$. In one dimension, this is a shielding approximation where the annihilation of the pair of particles $A_{3}$ and $A_{2}$ is neglected compared to the annihilation of the pair $A_{3} A_{1}$ when the three particles are aligned in the order $A_{3} A_{1} A_{2}$. On the other hand, in the half-space $E_{1}$ the distance $r^{\prime \prime}$ is greater than $r$ and Eq. (3) can be written as

$$
\rho_{3}\left(r, r^{\prime}, r^{\prime \prime}\right) \simeq \rho^{3}(t) X(r, t) X\left(r^{\prime}, t\right)=\gamma(r, t) \rho_{2}\left(r^{\prime}, t\right),
$$

where we define $\gamma(r, t)$ according to

$$
\gamma(r, t)=\rho(t) X(r, t)=\rho_{2}(r, t) / \rho(t) .
$$

Thus

$$
\begin{aligned}
& 2 \int_{E_{1}} \rho_{3}\left(r, r^{\prime}, r^{\prime \prime}\right) w\left(r^{\prime}\right) D^{d} r^{\prime} \\
& \quad \simeq \gamma(r, t) \int \rho_{2}\left(r^{\prime}, t\right) w\left(r^{\prime}\right) D^{d} r^{\prime},
\end{aligned}
$$

which from Eq. (1) is just $-\gamma(r, t) \partial \rho(t) / \partial t$. Finally in $E(r)$, as $r$ is large, in Eq. (3) the correlation $X(r, t)$ is unity at the leading order and one can use the approximation

$$
\rho_{3}\left(r, r^{\prime}, r^{\prime \prime}\right) \simeq \rho^{3}(t) X\left(r^{\prime}, t\right) X\left(r^{\prime \prime}, t\right)=\rho(t) \gamma\left(r^{\prime}, t\right) \gamma\left(r^{\prime \prime}, t\right) .
$$

The evolution equation Eq. (2) then becomes

$$
-\frac{\partial \rho_{2}}{\partial t}(r, t)=w(r) \rho_{2}(r, t)-\gamma(r, t) \frac{d \rho}{d t}(t)+\rho(t) I_{d}(r, t),
$$

where the integral $I_{d}(r, t)$ is restricted to region $E(r)$

$$
I_{d}(r, t)=2 \int_{E(r)} w\left(r^{\prime}\right) \gamma\left(r^{\prime}, t\right) \gamma\left(r^{\prime \prime}, t\right) D^{d} r^{\prime} .
$$

Expressed in terms of $\gamma(r, t)$, Eq. (6) has the following simpler form where the density has been eliminated:

$$
-\frac{\partial \gamma}{\partial t}(r, t)=w(r) \gamma(r, t)+I_{d}(r, t) .
$$

To end this section we compute explicitly $I_{d}(r, t)$ for the dimensions $d=1,2$, and 3 . In one dimension, $E(r)$ is the interval $\left[A_{1}, A_{2}\right]$, and $A_{3}$ is between $A_{1}$ and $A_{2}$, i.e., $r=r^{\prime}$ $+r^{\prime \prime}$. This gives a convolution integral

$$
I_{1}(r, t)=2 \int_{0}^{r} w\left(r^{\prime}\right) \gamma\left(r^{\prime}, t\right) \gamma\left(r-r^{\prime}, t\right) d r^{\prime} .
$$

In two dimensions, using the coordinates $A_{1}=(0,0), A_{2}$ $=(r, 0), A_{3}=\left(r^{\prime} \cos \theta, r^{\prime} \sin \theta\right)$ the domain $E(r)$ is the strip $\left[-\pi / 2 \leqslant \theta \leqslant \pi / 2,0 \leqslant r^{\prime} \leqslant r / \cos \theta\right]$ and

$$
I_{2}(r, t)=4 \int_{0}^{\pi / 2} d \theta \int_{0}^{r / \cos \theta} w\left(r^{\prime}\right) \gamma\left(r^{\prime}, t\right) \gamma\left(r^{\prime \prime}, t\right) r^{\prime} d r^{\prime},
$$

where

$$
r^{\prime \prime}=\left[r^{2}+r^{\prime 2}-2 r r^{\prime} \cos \theta\right]^{1 / 2} .
$$

In three dimensions, using the frame where $A_{1}=(0,0,0)$, $A_{2}=(0,0, r)$, and $A_{3}=\left(r^{\prime} \sin \theta \cos \varphi, r^{\prime} \sin \theta \sin \varphi, r^{\prime} \cos \theta\right)$ the region $E(r)$ is given by $\left[0 \leqslant \varphi \leqslant 2 \pi, 0 \leqslant \theta \leqslant \pi / 2,0 \leqslant r^{\prime}\right.$ $\leqslant r / \cos \theta]$ and finally

$I_{3}(r, t)$

$$
=4 \pi \int_{0}^{\pi / 2} \sin \theta d \theta \int_{0}^{r / \cos \theta} w\left(r^{\prime}\right) \gamma\left(r^{\prime}, t\right) \gamma\left(r^{\prime \prime}, t\right) r^{\prime 2} d r^{\prime},
$$

where $r^{\prime \prime}$ is again given by the Eq. (11). We thus shall use the representation

$I_{d}(r, t)$

$$
=\int_{0}^{\pi / 2} \lambda_{d}(\theta) d \theta \int_{0}^{r / \cos \theta} w\left(r^{\prime}\right) \gamma\left(r^{\prime}, t\right) \gamma\left(r^{\prime \prime}, t\right) r^{\prime(d-1)} d r^{\prime},
$$


where $\lambda_{1}=2 \delta(\theta) \quad$ (the Dirac function), $\lambda_{2}=4, \quad \lambda_{3}$ $=4 \pi \sin \theta$, and $r^{\prime \prime}$ given by Eq. (11). Explicit solutions $\gamma(r, t)$ of Eq. (8) where $I_{d}(r, t)$ has the form given in Eq. (13) are studied in the next section.

\section{SCALING FORM OF THE EVOLUTION EQUATION}

The reaction radius $R(t)$, defined by $t w(R(t))=1$, i.e., $R(t)=r_{0} \ln t$ when $w(r)=e^{-r / r_{0}}$, is such that at large time no particles survive if their separation $r$ is smaller than $R(t)$. In this regime it is thus convenient to use a scaled variable $x=r / R(t)$ and to see if Eq. (8) admits asymptotic solutions of the form

$$
\gamma(r, t)=\rho(t) X(r, t)=h(x) / R^{\alpha}(t) .
$$

Imposing the condition $X(r \rightarrow \infty, t)=1$ in the previous definition gives the density and the pair correlation function in term of $h(x)$ according to

$$
\begin{gathered}
\rho(t)=\lim _{x \rightarrow \infty} h(x) / R^{\alpha}(t), \\
X(r=x R(t), t)=h(x) / h(\infty) .
\end{gathered}
$$

These expressions evidently imply that $h(x)$, which is expected to vanish on some interval $0 \leqslant x<x_{1}$, is elsewhere positive and bounded. To study $h(x)$, we begin by inserting Eq. (14) into Eq. (8). Since $\partial x / \partial t=-x / t \ln t$, one easily finds

$$
-\frac{\partial \gamma}{\partial t}(r, t)=x^{1-\alpha} \frac{d}{d x}\left[x^{\alpha} h(x)\right] / t R^{\alpha}(t) \ln t .
$$

To express the integral $I_{d}(r, t)$ we change the variables according to $r=x R(t), r^{\prime}=y R(t)$, and $r^{\prime \prime}=z R(t)$ where from Eq. (11) $z$ is defined by

$$
z=\left[x^{2}+y^{2}-2 x y \cos \theta\right]^{1 / 2} \text {. }
$$

Thus $\quad \gamma\left(r^{\prime}, t\right) \gamma\left(r^{\prime \prime}, t\right)=h(y) h(z) R^{-2 \alpha}(t) \quad$ and $\quad I_{d}(r, t)$ $=R^{d-2 \alpha}(t) J_{d}(x, t)$ with

$$
J_{d}(x, t)=\int_{0}^{\pi / 2} \lambda_{d}(\theta) d \theta \int_{0}^{x / \cos \theta} t^{-y} h(y) h(z) y^{d-1} d y,
$$

where we have used the relation $w(y R(t))=t^{-y}$, which also gives $w(r) \gamma(r, t)=t^{-x} h(x) R^{-\alpha}(t)$. If we multiply both sides of Eq. (8) by $t R^{\alpha}(t) \ln t$, we obtain

$$
x^{1-\alpha} \frac{d}{d x}\left[x^{\alpha} h(x)\right]=t^{1-x} h(x) \ln t+t R^{d-\alpha}(t) J_{d}(x, t) \ln t .
$$

The left-hand side of the previous equation is time independent, and this has to be the case for each member of the right-hand side, since they are non-negative. The first term implies that

$$
h(x)=0 \text { for } 0 \leqslant x \leqslant 1 .
$$

For the second term, one can observe that for any function $H(y)$ that vanishes for $0 \leqslant y<y_{1}<y_{2}$, and such that $H\left(y_{1}\right)$ $\neq 0$, then at large time

$$
\int_{0}^{y_{2}} t^{-y} H(y) d y=t^{-y_{1}} \ln ^{-1} t\left[H\left(y_{1}\right)+O\left(\ln ^{-1} t\right)\right] .
$$

Applying this relation to the $y$-integration appearing in Eq. (19) gives for the leading behavior of $J_{d}(x, t), J_{d}(x, t)$ $\simeq C(x) t^{-y_{1}} \ln ^{-1} t$ and thus the second term of the right-hand side of Eq. (20) behaves according to $t^{1-y_{1}} R^{d-\alpha}(t) C(x)$, $C(x)$ being a positive time-independent function and $y_{1}$ the smallest value for which $h(y)$ is strictly positive. Time independence of this term thus requires that $y_{1}=1$, in agreement with Eq. (21) and the physical interpretation of the reaction radius, and we deduce

$$
\alpha=d
$$

For $x \geqslant 1$, we finally obtain the following equation to determine the function $h(x)$ :

$$
\begin{aligned}
x^{1-d} \frac{d}{d x}\left[x^{d} h(x)\right]= & h(1) \int_{0}^{\pi / 2} \lambda_{d}(\theta) h \\
& \times\left(\left[1+x^{2}-2 x \cos \theta\right]^{1 / 2}\right) d \theta .
\end{aligned}
$$

Among the solutions of Eq. (24) we have to select the bounded ones which can fulfill Eq. (15) and (16). This constraint determines $h(1)$, since equating the two members of Eq. (24) in the limit $x \rightarrow \infty$ one obtains for a bounded solution $d h(\infty)=h(1) h(\infty) \int_{0}^{\pi / 2} \lambda_{d}(\theta) d \theta$. These values are

$$
\begin{gathered}
d=1, \quad h(1)=1 / 2, \quad d=2, \quad h(1)=1 / \pi, \\
d=3, \quad h(1)=3 / 4 \pi .
\end{gathered}
$$

Finally, Eq. (24) becomes for $x>1$

$x^{1-d} \frac{d}{d x}\left[x^{d} h(x)\right]=\int_{0}^{\pi / 2} \mu_{d}(\theta) h\left(\left[1+x^{2}-2 x \cos \theta\right]^{1 / 2}\right) d \theta$,

with $\mu_{1}(\theta)=\delta(\theta), \mu_{2}(\theta)=4 / \pi$, and $\mu_{3}(\theta)=3 \sin \theta$. We obtain the solution of this equation in the next section.

\section{DETERMINATION OF THE SCALING SOLUTIONS}

In one dimension, we have to solve

$$
\frac{d}{d x}[x h(x)]=h(x-1), \quad h(1)=1 / 2 .
$$

Since $h(x)$ vanishes for $0 \leqslant x<1$, this equation determines $h(x)$ on intervals of unit length and at each step we require continuity at integer values of $x$. For example, on the first few intervals one gets 


$$
\begin{gathered}
1 \leqslant x \leqslant 2, \quad h(x)=1 / 2 x, \\
2 \leqslant x \leqslant 3, \quad h(x)=[1+\ln (x-1)] / 2 x
\end{gathered}
$$

and one observes that $h(x)$ is close to its asymptotic value as soon as $x \gtrsim 2.5$. For example one obtains $h(2.5)=0.281$, $h(3)=0.282$, the exact value being

$$
h(\infty)=e^{-\gamma / 2} \simeq 0.2807,
$$

where $\gamma \simeq 0.57721 \ldots$ is the Euler constant. This result can be obtained in the following way. Let $H(p)$ be the Laplace transform of $h(x), H(p)=\int_{1}^{\infty} e^{-p x} h(x) d x$. From Eq. (27), it fulfills

$$
p \frac{d}{d p} H(p)+e^{-p} H(p)+e^{-p} / 2=0,
$$

whose solution is $H(p)=\left\{\exp \left[E_{1}(p)\right]-1\right\} / 2$, where $E_{1}(p)$ is the exponential integral

$$
E_{1}(p)=\int_{p}^{\infty} e^{-y} d y / y \simeq-\gamma-\ln p \quad \text { as } p \rightarrow 0 .
$$

The asymptotic value given in Eq. (29) then follows from the usual relation $h(\infty)=\lim [p H(p), p \rightarrow 0]$ These results, which we have already derived [7] in a slightly different way, imply a density and a pair correlation function in perfect agreement with the MC simulations of the annihilation process, as shown in Ref. [10].

In higher dimensions, for $x>1$ we have to solve the equation

$$
\begin{aligned}
h(x)= & x^{-d}\left[h(1)+\int_{1}^{x} y^{d-1} \int_{0}^{\pi / 2} \mu_{d}(\theta) h\left(\left[1+y^{2}\right.\right.\right. \\
& \left.\left.-2 y \cos \theta]^{1 / 2}\right)\right] d \theta d y
\end{aligned}
$$

and we are unable to find an analytic expression for $h(x)$. However, the basic features of the one-dimensional solution persist: it varies significantly only on the range $1<x<2$, its asymptotic value $h(\infty)$ being practically reached for $x$ $\simeq 2.5$. These facts appear easily when Eq. (32) is solved numerically by iterations. We obtain

$$
d=2, \quad h(\infty) \cong 0.188, \quad d=3, \quad h(\infty) \cong 0.144,
$$

which through Eq. (15) give the asymptotic density. These values are in agreement with the MC results already reported in [10] from the work of [12], which correspond to

$$
d=2, \quad 0.18 \leqq h(\infty) \lesssim 0.21, \quad d=3, \quad 0.14 \lesssim h(\infty) \leqq 0.17 .
$$

Our values (33) are close to the lower limits in Eq. (34), but this is expected since in the simulations the density is
TABLE I. Values of the asymptotic correlation $X_{\infty}(x)$ for $x$ in the range $[1,3]$. The columns corresponding to $d=1$ and $d=3$ are the predicted values. The two columns for $d=2$ are the theoretical predictions (first column) and the measured values (second column) in $\mathrm{MC}$ simulations.

\begin{tabular}{ccccc}
\hline \hline$x$ & $d=1$ & \multicolumn{2}{c}{$d=2$} & $d=3$ \\
\hline 1.0 & 1.78 & 1.69 & $1.40(4)$ & 1.66 \\
1.1 & 1.62 & 1.48 & $1.42(3)$ & 1.39 \\
1.2 & 1.48 & 1.32 & $1.32(3)$ & 1.24 \\
1.3 & 1.37 & 1.21 & $1.22(3)$ & 1.15 \\
1.4 & 1.27 & 1.12 & $1.15(3)$ & 1.06 \\
1.5 & 1.19 & 1.05 & $1.05(3)$ & 1.02 \\
1.6 & 1.11 & 1.01 & $1.03(3)$ & 0.99 \\
1.7 & 1.05 & 0.97 & $1.00(3)$ & 0.98 \\
1.8 & 0.99 & 0.95 & $0.95(3)$ & 0.97 \\
1.9 & 0.94 & 0.94 & $0.92(3)$ & 0.98 \\
2.0 & 0.89 & 0.96 & $0.95(3)$ & 0.99 \\
2.2 & 0.96 & 0.99 & $1.00(3)$ & 1.00 \\
2.4 & 0.99 & 1.00 & $1.00(3)$ & 1.00 \\
2.6 & 1.01 & 1.00 & $1.00(3)$ & 1.00 \\
2.8 & 1.01 & 1.00 & $1.00(3)$ & 1.00 \\
3.0 & 1.00 & 1.00 & $1.00(3)$ & 1.00 \\
\hline \hline
\end{tabular}

overestimated from the finite size effects (free boundary conditions are used and some particles artificially survive). The values given in Eq. (33), together with our findings for 1 $\leqslant x \leqslant 3$, are used to predict the asymptotic correlation $X_{\infty}(x)=h(x) / h(\infty)$ given in Table I. Its values for $d=1$ have been already compared with the MC results in [10], and we add in this table the $d=2 \mathrm{MC}$ data taken from the Ref. [12]. These data, corresponding to $R(t)=14$, are in good agreement with our predictions, except in the vicinity of the discontinuity for $x=1^{+}$, where the full jump is truly asymptotic.

\section{CONCLUSION}

The agreement of the MC data with our results indicate, as expected, that the Kirkwood superposition approximation gives a precise description of the static annihilation process. We have shown in this work the particular scaling form of the large time limit of the density and the correlation implied by this assumption. It must be stressed, as it is clear from our derivation, that the asymptotic regime is independent of the initial density. The self-organized effects that we find decrease smoothly as the dimensionality of the system increases, the $d=\infty$ limit being mean-field-like, with $X_{\infty}(r)$ $=\theta(r-R(t))$.

To conclude, we want to mention that the analysis we have performed here can be easily extended to the annihilation $A+B \rightarrow 0$, with $\alpha=d / 2$, and also extended to others forms of the annihilation.

\section{ACKNOWLEDGMENTS}

I thank Dr. R. Brown and Dr. Y. Meurdesoif for communicating to me the MC data, and for their interest in this work. 
[1] G. Oshanin, S. Burlatsky, E. Clement, D. S. Graff, and L. M. Sander, J. Phys. Chem. 98, 7390 (1994).

[2] N. A. Efremov, S. G. Kulikov, R. I. Personov, and Yu. V. Romanovskii, Chem. Phys. 128, 9 (1988).

[3] H. Schnörer, V. Kuzovkov, and A. Blumen, J. Chem. Phys. 92, 2310 (1990).

[4] B. Bonnier and E. Pommiers, Phys. Rev. E 52, 5873 (1995).

[5] M. Tachiya and A. Mozumder, Chem. Phys. Lett. 28, 87 (1974).
[6] S. F. Burlatsky and A. I. Cheroustan, Phys. Lett. 56A, 145 (1990).

[7] B. Bonnier and R. Brown, Phys. Rev. E 55, 6661 (1997).

[8] V. Kuzovkov and E. Kotomin, Rep. Prog. Phys. 51, 1479 (1988).

[9] H. Schnörer, V. Kuzovkov, and A. Blumen, Phys. Rev. Lett. 63, 805 (1989).

[10] B. Bonnier, R. Brown, and E. Pommiers, J. Phys. A 28, 5165 (1995).

[11] J. G. Kirkwood, J. Chem. Phys. 3, 300 (1935).

[12] R. Brown and N. A. Efremov, Chem. Phys. 155, 357 (1991). 